\title{
Effect of Starch Oxidation Degree on the Properties of Hydrogels from Dialdehyde Starch and Polyvinyl Alcohol
}

\author{
Jahel Desire Carrera ${ }^{1}$, Daniela Alejandra Viteri Narváez¹, Marco Leon², José Francisco Alvarez-Barreto*,1 \\ ${ }^{1}$ Universidad San Francisco de Quito, Department of Chemical Engineering, Institute for the Development of Alternative Energies and \\ Materials, IDEMA, Biomaterials Laboratory, 170901, Ecuador \\ ${ }^{2}$ Universidad San Francisco de Quito, Department of Mechanical Engineering, 170901, Ecuador
}

A R T I C L E I N F O

Article history:

Received: 30 September, 2020

Accepted: 28 November, 2020

Online: 16 December, 2020

Keywords:
Dialdehyde starch
Hydrogels
Polyvinyl alcohol
Swelling
Controlled drug release

Keywords:

Dialdehyde starch

Polyvinyl alcohol

Controlled drug release

\begin{abstract}
A B S T R A C T
Starches have been applied as biomaterials due to their wide availability and biocompatibility. These have also been modified by oxidation, resulting in dialdehyde starch (DAS), to improve their stability in water and mechanical properties. Cassava starch with a low oxidation degree has been introduced into hydrogels based on polyvinyl alcohol (PVA) to improve their properties. However, the behavior of these materials with starch at different oxidation levels has not been previously explored. In the present work, the effect of the oxidation degree of cassava starch on the physical and chemical properties of DASPVA hydrogels was evaluated. To modify the degree of oxidation, different concentrations of $\mathrm{H}_{2} \mathrm{O}_{2}$ were used, and a high degree of oxidation was achieved by incorporating copper sulfate II as a catalyst. Oxidation was confirmed by quantification of carbonyl groups and Fourier Transformed Infrared Spectroscopy. Hydrogels with low and medium oxidation $D A S$ displayed greater swelling, but also lower stability over time. Similarly, scanning electron microscopy confirmed greater porosity in them. On the other hand, hydrogels with high oxidation DAS had lower water absorption capacity, but greater stability over time. Regarding the controlled release of ibuprofen, as a model drug, hydrogels formulated with low and medium oxidation DAS presented a greater and faster release, compared to the formulations with high oxidation DAS. These results showed that the degree of starch oxidation, for the PVA-DAS hydrogel synthesis has a significant effect on the behavior of the polymeric network.
\end{abstract}

\section{Introduction}

Hydrogels are three-dimensional polymeric networks that are capable of containing significant amounts of water. They have been used in biomedical applications, such as tissue engineering and controlled drug release [1]. Furthermore, hydrogels have tunable biocompatibility, and respond to external stimuli such as $\mathrm{pH}$, temperature, and concentrations of species, among others [2]. Thus, their porous structures are suitable for the encapsulation of drugs for a subsequent controlled release [3].

Hydrogels can be synthetized from virtually any water-soluble polymer, either natural or synthetic, such as alginic acid, pectin, carrageenan, dextran sulfate, chitosan, poly-lysine, and agarose [4]. Poly(vinyl) alcohol (PVA), is a hydrophilic, synthetic

${ }^{*}$ Corresponding Author: José Francisco Alvarez-Barreto, Email:

jalvarezb@usfq.edu.ec

www.astesj.com

https://dx.doi.org/10.25046/aj0506165 biodegradable polymer that has been widely used, due to its proved biocompatibility [5]. An additional benefit is that this polymer can be crosslinked physically, without the need of potentially toxic chemical agents . This can be achieved through freezing / thawing cycles, where the polymer in aqueous solution depending on its concentration and freeze cycles, form polymer microcrystals [1]. Nevertheless, since it is a petroleum derivative, PVA has limited availability. At the same time, in order to obtain stable structures, PVA concentrations must be high, up to $35 \% \mathrm{w} / \mathrm{v}$, with molecular weights of $98000 \mathrm{Da}$ or greater [2]. Therefore, its use poses an economical limitation for the creation of new technologies, especially in developing countries.

Starch, on the other hand, is one of the most abundant and cheapest polysaccharides [6]. It is comprised of two main molecules: amylose, a linear macromolecule composed of 250-300 
$\alpha(1 \rightarrow 4)$-linked-D-glucose, amylopectin, a less hydrophilic and branched macromolecule, and constitutes about $80 \%$ of the granular structure. To form starch hydrogels, it is possible to chemically modify its structure in order to have a greater number of functional groups [4,7]. Oxidation, for example, breaks the long glucose chains of the polymer molecules, replacing the hydroxyl groups for carbonyl and carboxyl groups. This results in reduced viscosity [8]. In drug-release applications, integrity and stability in a humid environment are required over a relatively long period of time. It has been shown that the use of dialdehyde starch (DAS), resulting from oxidation, exhibits a substantially lower solubility in water, making it more suitable for this application [9].

In [9], the authors developed protein-based films with dialdehyde starch (DAS), PVA and feather keratin. They found that if DAS was not added, an increase of the PVA concentration in the blended films resulted in a slight decrease of the film's solubility in water. By adding $5 \%$ of DAS, the total solubility mass of the film was reduced, which indicates an increase in the degree of cross-linking.

The most commonly used modifier in starch oxidation is hypochlorite, but despite being chemically efficient, it results in the formation of chlorinated products, that could potentially be toxic [10]. Several comparative studies have been carried out on the properties of starches at different degrees of oxidation with hypochlorite[10]; nevertheless, no comparative study is found in the literature on the effect that hydrogen peroxide has on different degrees of oxidation in cassava starch, and thereby in the formulations of hydrogels [11].

In [12], the authors synthesized hydrogels based on PVA and modified cassava starch as carriers for controlled drug delivery. In this research, the oxidation of starch was done with sodium hypochlorite solution containing $5 \%$ active chlorine. The hydrogels synthetized with DAS/PVA, showed an enhanced stability in water; nevertheless, the oxidation degree was maintained constant through the entire research. However, it has been demonstrated that, for other biomaterial applications, starch oxidation degree has important implications, such as bioplastics, in material behavior $[13,14]$. For this reason, the present study proposes to determine the effects of the oxidation degree of cassava starch on the physical and chemical properties of DASPVA hydrogels.

\section{Materials and Methods}

\subsection{Chemical materials}

Cassava starch was obtained from Ecuador's local stores. Hydroxylamine hydrochloride (ACS Reagent Grade, 5470-11, ACROS Organics), Poly vinyl alcohol (PVA, Mowiol 28-99, MW 145000, 99.0-99.8 mol\% hydrolysis, Millipore Sigma, Cat. 10849), Sodium Phosphate, Dibasic, Anhydrous (3828-01, J.T.Baker), Potassium chloride (6858, Mallinckrodt), di-Potassium hydrogen phosphate, anhydrous GR for analysis (1.05104.1000, MERCK), Ibuprofen ( $\geq 98 \%$ GC, Millipore Sigma, Cat. I4883) were used as received.

\subsection{Starch Oxidation}

Starch oxidation was performed with hydrogen peroxide $\left(\mathrm{H}_{2} \mathrm{O}_{2}\right)$, through adaptation of the methodology proposed by [15].
Briefly, $\mathrm{H}_{2} \mathrm{O}_{2}$ solutions at 3,5 and $10 \% \mathrm{v} / \mathrm{v}$ were prepared in distilled water. For the chemical modification, a $2 \% \mathrm{w} / \mathrm{v}$ solution of starch in distilled water was gelatinized at $80^{\circ} \mathrm{C}$ for 30 minutes, under moderate stirring. Later, the solution was cooled until room temperature, and its $\mathrm{pH}$ was adjusted to 7 . For every $2 \mathrm{~g}$ of gelatinized starch, $6.25 \mathrm{~mL}$ of $\mathrm{H}_{2} \mathrm{O}_{2}$ solution were added, drop wise during $1 \mathrm{~h}$ at $25^{\circ} \mathrm{C}$, under continuous stirring, maintaining $\mathrm{pH}$ at 7.0. When the oxidation process was done, an equal volume of $70 \% \mathrm{v} / \mathrm{v}$ ethanol was added, and starch precipitation was allowed for 1 hour. The supernatant was discarded, and the remaining mixture was centrifuged at $1000 \mathrm{~g}$ for 5 minutes. Five rinsing cycles were carried out with $\mathrm{diH}_{2} \mathrm{O}$, in a similar fashion. The resulting dialdehyde starch (DAS) was dried at $40^{\circ} \mathrm{C}$ until constant weight.

To obtain a high degree of oxidation DAS, copper (II) sulfate was used as a catalyst, as proposed by [16]. Starch was gelatinized with the same methodology described above. Before adding the $\mathrm{H}_{2} \mathrm{O}_{2}$ solution at $10 \% \mathrm{v} / \mathrm{v}$, the catalyst was incorporated at $0.1 \%$ $\mathrm{w} / \mathrm{v}$, and the reaction was allowed to take place for 1 hour at $25^{\circ} \mathrm{C}$. Subsequently, precipitation and washing were done as previously mentioned.

\subsection{Starch characterization}

Carbonyl groups were quantified based on the method proposed by [17], with some modifications. Briefly, $1 \mathrm{~g}$ of DAS was mixed with $25 \mathrm{~mL}$ of distilled water, gelatinized at $80^{\circ} \mathrm{C}$ under constant stirring, and then, cooled at room temperature. The $\mathrm{pH}$ was adjusted to 3.2 , and, immediately, $3,75 \mathrm{~mL}$ of hydroxylamine hydrochloride $(25 \mathrm{~g}$ of hydroxylamine hydrochloride in $100 \mathrm{~mL}$ of $\mathrm{NaOH} 0.5 \mathrm{M}$ in a $500 \mathrm{~mL}$ solution) were added to the gelatinized solution, with subsequent incubation at $40^{\circ} \mathrm{C}$ for $4 \mathrm{~h}$. A potentiometric titration followed, with $0.1 \mathrm{M} \mathrm{HCl} \mathrm{until} \mathrm{pH} 3.2$. The amount of carbonyl groups per 100 glucose units (CO/100GU), was calculated through equation 1 :

$$
\frac{C O}{100 G U}=\frac{(V b-V s) \times M \times 0,028 \times 100}{W}
$$

where, $\mathrm{Vb}$ and $\mathrm{Vs}$ are the volumes $(\mathrm{mL})$ of $\mathrm{HCL}$ used to titrate the blank (native starch) and the oxidized starch, respectively; $M$ is the molar concentration of $\mathrm{HCl}(\mathrm{mol} / \mathrm{L})$, and $\mathrm{W}$ is the weight of the sample on a dry basis $(\mathrm{g})$.

On the other hand, carboxyl groups were determined using the protocol proposed by [15]. Briefly, 1g of DAS was suspended in $60 \mathrm{~mL}$ of distilled water, gelatinized and cooled, as previously mentioned. Later, a potentiometric titration took place until $\mathrm{pH} 8.2$, using $\mathrm{NaOH} 0.01 \mathrm{M}$. The amount of carboxyl groups per 100 glucose units $(\mathrm{CO} / 100 \mathrm{GU})$, was calculated through equation 2 :

$$
\frac{\mathrm{COOH}}{100 \mathrm{GU}}=\frac{(\mathrm{Vs}-\mathrm{Vb}) \times M \times 0,045 \times 100}{W}
$$

where, $V s$ and $V b$ are the titration volumes $(\mathrm{mL})$ of the oxidized starch and the blank (native starch), respectively; $M$ is the molarity of $\mathrm{NaOH}(\mathrm{mol} / \mathrm{L})$, and $W$ is the sample weight $(\mathrm{g})$.

\subsection{Hydrogel preparation}

Hybrid hydrogels with Polyvinyl Alcohol (PVA) and dialdehyde starch were synthetized through a freezing/thawing method, by adaptation of the protocol reported by [2]. The 
common protocol for the preparation was to completely dissolve PVA in distillated water under continuous stirring at $95^{\circ} \mathrm{C}$, at $20 \%$ w/v. In parallel, a starch suspension, at a given concentration, was gelatinized as previously mentioned. Both solutions were then mixed at a 1:1 ratio in a vortex homogenizer, with alternation with heating in a water bath at $70{ }^{\circ} \mathrm{C}$, for a total time of $30 \mathrm{~min}$. Then, 5 $\mathrm{mL}$ of the polymeric mixture were poured into 1-inch petri-dishes.

Crosslinking was induced through freezing/thawing cycles; the polymeric mixture in the petri dish was frozen at $-20^{\circ} \mathrm{C}$ for 12 $\mathrm{h}$, followed by thawing at $25^{\circ} \mathrm{C}$ for $3 \mathrm{~h}$. Table 1 specifies the variables that were modified for the different conditions of hydrogel preparation in order to identify the formulations that could be suitable for the subsequent ibuprofen encapsulation and release. It was decided to vary the number of freeze/thawing cycles because through every cycle, there is a formation of microcrystals through the polymeric network, allowing a high crosslinking degree. Consequently, after thawing, the mixture was frozen again, and this process was repeated according to the chosen number of cycles. Since one of the objectives of this research is to partially substitute the amount of PVA in hydrogels formulations, the final concentration of the latter was maintained constant at $10 \% \mathrm{w} / \mathrm{v}$. Hydrogels with native starch were used as controls.

Table 1: Variables for the synthesis of hybrid DAS/PVA hydrogels

\begin{tabular}{|c|c|c|c|}
\hline Variable & \multicolumn{3}{|c|}{ Values (nomenclature) } \\
\hline $\begin{array}{c}\text { Oxidation degree } \\
\left(\% \mathrm{H}_{2} \mathrm{O}_{2}\right)\end{array}$ & 3 & 5 & $10,10^{*}$ \\
$(3 \mathrm{DAS})$ & $(5 \mathrm{DAS})$ & $(10 \mathrm{DAS}$, \\
& & & $10 \mathrm{DASC})$ \\
\hline $\begin{array}{c}\text { DAS concentration }(\% \\
\text { w/v) }\end{array}$ & 5 & 10 & 15 \\
\hline Crosslinking cycles & 1 & 2 & 3 \\
\hline
\end{tabular}

*Copper (II) sulfate was added to obtain high oxidation DAS

According to the protocol described by [18], after crosslinking, hydrogels were cut in $5 \mathrm{~mm}$ diameter discs, and placed in 24-well plates with $2 \mathrm{~mL}$ of $1 \mathrm{X}$ phosphate buffered saline (PBS, pH 7.3), in an incubator, at $37^{\circ} \mathrm{C}$. The hydrogel weight was registered at 0, 5, 15, $60 \mathrm{~min}, 2,3,4,5,24,48$ and $72 \mathrm{~h}$. Wateruptake was calculated according to equation 3 .

$$
\text { Water-uptake }(\%)=\frac{\left(W_{t}-W_{1}\right)}{W_{1}} \times 100
$$

where $\mathrm{W}_{1}$ is the initial weight of the hidrogel, and $\mathrm{W}_{\mathrm{t}}$ its weight at a given time.

\subsection{FTIR Analysis}

Analyses were conducted in a Fourier transformed infrared spectrometer; model Cary 630 FTIR-ATR (Agilent Technologies) for the native and oxidized starches, as well as for the different hydrogel formulations [15]. Spectra were acquired in the region of $4000-500 \mathrm{~cm}-1$.

\subsection{Scanning Electron Microscopy (SEM)}

Hydrogel morphological analyses were carried out in a JEOL JSM-IT300 Scanning Electron Microscope (Tokyo, Japan) with lyophilized hydrogels. Samples were cut and placed on metallic stubs with carbon tape. Images were obtained at $5 \mathrm{kV}$ and $50 \mathrm{~Pa}$, at different magnifications.

\subsection{Ibuprofen encapsulation and release}

Ibuprofen was incorporated into the hydrogels at a concentration of $10 \mathrm{mg} / \mathrm{mL}$, as proposed by [18], by adding the drug into the PVA solution at $50^{\circ} \mathrm{C}$. The same process described above for hydrogel preparation was followed. The release was performed as previously described [18]. DAS/PVA hydrogels with ibuprofen were cut into $5 \mathrm{~mm}$ diameter and placed in $2 \mathrm{~mL}$ of PBS at $37^{\circ} \mathrm{C}$. Later, $400 \mu \mathrm{L}$ of supernatant were collected and replaced with fresh PBS at 15, 30, 60, 120, 180, 240, 300, $360 \mathrm{~min}, 24,48$ and $72 \mathrm{~h}$. Four samples of each hydrogel formulation were tested for Ibuprofen release.

Each supernatant was placed in $2.6 \mathrm{~mL}$ of PBS, in a quartz cuvette, and the optical density of this final solution was determined at $222 \mathrm{~nm}$ in a CECIL CE 2041 UV-VIS spectrophotometer. The sample concentration was calculated with a calibration curve done with known solutions of ibuprofen in PBS. The release kinetics was expressed as the percent cumulative release of ibuprofen over time.

\subsection{Statistical analyses}

All assays were performed with three samples of each formulation, and four in the case of ibuprofen release. The results are expressed as the average \pm standard deviation. A two-way analysis of variance (ANOVA) was also applied, and multiple pairwise comparisons were carried out using Turkey-HSD, with a $95 \%$ confidence level $(\mathrm{p}<0.05)$.

\section{Results and discussion}

\subsection{Oxidized starch characterization}

Carbonyl and carboxyl contents of native and oxidized starches (dialdehyde starch, DAS) with low and high degrees of oxidation are shown in Table 2. It can be observed that carbonyl was the primary functional group produced during the oxidation process, whereas, a significantly minor amount of carboxyl groups was formed. It has been reported that the reaction mechanism of hydrogen peroxide with starch proceeds via a radical chain reaction [19] . It is more evident in dialdehyde catalyzed starch with copper sulfate, since, in the presence of the metal catalyst, the $\mathrm{H}_{2} \mathrm{O}_{2}$ is decomposed into a hydroxyl radical. Then, this radical rapidly reacts with the carbohydrate by eliminating hydrogen from a $\mathrm{C}-\mathrm{H}$ group on the sugar ring, forming a $\mathrm{R} * \mathrm{CHOH}$ radical, which further undergoes acid or base catalyzed rearrangement resulting in the cleavage of the glycosidic bond and formation of a carbonyl group [19].

One assumption that the authors in [19] proposed, is that the reaction between starch and hydrogen peroxide is so rapid that the major quantity of reagent was consumed for hydrogen abstraction, affording high amounts of carbonyl groups during the early period of time; therefore, less oxidant was available for the oxidation of 
carbonyl to carboxyl groups. At the same time, the reaction time on the current research was stablished at one hour; which is in accordance with the literature since it has been shown that the oxidation of hydroxyl groups in starch molecules by $\mathrm{H}_{2} \mathrm{O}_{2}$ was almost completed within the first 30 minutes.

Table 2: Carbonyl and carboxyl contents of dialdehyde cassava starch at different oxidation levels

\begin{tabular}{|c|c|c|}
\hline $\begin{array}{c}\mathrm{H}_{2} \mathrm{O}_{2} \\
\text { Concentration } \\
(\% \mathbf{\%} / \mathbf{v})\end{array}$ & $\begin{array}{c}\text { Carbonyl content } \\
{[\mathbf{C O} / 100 \text { GU] }}\end{array}$ & $\begin{array}{c}\text { Carboxyl content } \\
{[\mathbf{C O} / 100 \text { GU] }}\end{array}$ \\
\hline 3 & $0.255 \pm 0.091$ & $0.046 \pm 0.010$ \\
\hline 5 & $0.419 \pm 0.124$ & $0.034 \pm 0.009$ \\
10 (Catalyzed) & $2.875 \pm 0.089$ & $0.025 \pm 0.003$ \\
\hline
\end{tabular}

GU: Glucose units

Oxidation degree is determined by carbonyl and carboxyl content. The relatively bulky carbonyl and carboxyl groups are introduced with a partial depolymerization of the starch chains; for this reason, the determination of these values shows the degree of oxidation degree. A major carbonyl content is an indication of a higher oxidation degree [20].In this study, a low degree of oxidation will be considered as having a carbonyl content of less than $1 \mathrm{CO} / 100 \mathrm{GU}$; thus, all starches oxidized without catalyst will be within this category.

\subsection{Hydrogel morphological analyses}

It was important to evaluate hydrogel morphology as it can have important implications in swelling behavior and controlled released, for which a porous structure is desired. Lyophilized hydrogels with the same compositions, but different DAS are presented in Figure 1. Natural porous materials have no uniform porosity; rather, it is distributed allowing to maximize the overall performance of the structure [21]. For drug delivery applications, a balance between adequate porosity and mechanical stability is required.. At comparing the different images, it is possible to recognize that hydrogels with low oxidation degree have flatter and larger pores; while, in high oxidation DAS, the pores seem to be smaller and rounded. There is clear indication that pore arrangement is altered by the incorporation of starch, and that the oxidation degree further influences pore morphology and structure. Additionally, PVA only hydrogels (without starch) appeared to less porous.

Hybrid hydrogels composed with hydrophilic-hydrophobic polymers have been reported previously in literature. It has been shown that one of the major issues in obtaining porosity is due to the intrinsic immiscibility of hydrophilic (i.e. PVA) and hydrophobic (i.e. DAS) polymers, which, in turn, affect pore structure, and potentially, distribution.

\subsection{FTIR analysis}

The Fourier transformed infrared absorption spectra of lyophilized hydrogels composed with $10 \% \mathrm{w} / \mathrm{v}$ PVA and $10 \% \mathrm{w} / \mathrm{v}$ starch is presented in Figure 2. In the case of FTIR spectra for different hydrogel formulations with oxidized starch, there is a decreased intensity. As previously reported in the literature, this lower intensity corresponds to crosslinked polymer structures, probably associated with a major bond vibration restriction [22]. A stretching band can be observed at $2850 \mathrm{~cm}^{-1}$ due to C-H broad alkyl. Another lower intensity stretching is because of the strong$\mathrm{OH}$ group band for free unreacted alcohol and hydrogen bonded bands at 3600-3200 cm-1, which are associated with freeze-thawed PVA [23]. As expected, the peak of dialdehyde starch appears at $1630 \mathrm{~cm}^{-1}-1710 \mathrm{~cm}^{-1}$, due to $\mathrm{C}=\mathrm{O}$ and $\mathrm{C}=\mathrm{C}$ stretching frequency groups that are present in DAS [24].
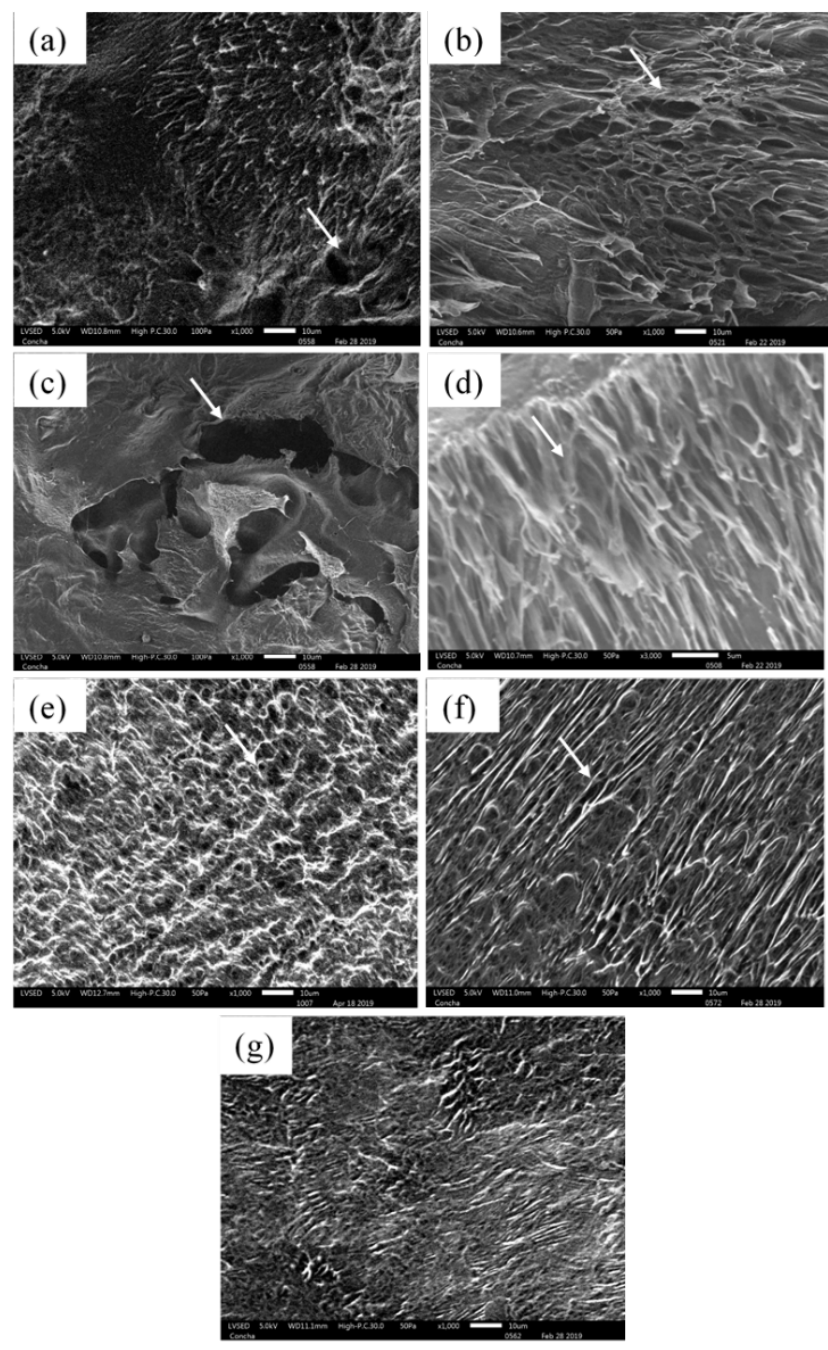

Figure 1: Scanning electron micrographs of lyophilized hydrogels composed of $10 \% \mathrm{w} / \mathrm{v}$ PVA, $10 \% \mathrm{w} / \mathrm{v}$ Starch and 1 crosslinking cycle: (a) NS, (b) 3DAS, (c) 5 DAS, (d) 10 DAS, (e) 10DASC, (f) Solely $20 \% \mathrm{w} / \mathrm{v}$ PVA and (g) Solely $10 \%$ w/v PVA. Calibration bar: $10 \mu \mathrm{m}$. Figure 1d was reported at 3000X (Calibration bar: $5 \mu \mathrm{m}$ ) in order to better observe the porous structure.

When comparing the spectra of hydrogels composed solely with PVA to hybrid hydrogels, there is lower intensity absorbance in the different bands; nevertheless, those associated with -OH groups, could be related to a strong interaction between both polymers forming a tight network, as suggested by [22]. PVA addition enhances the elasticity of hydrogels, which is corroborated by the increased intensity of the $\mathrm{C}-\mathrm{H}$ stretching 
absorption band at $2031 \mathrm{~cm}-1$, and the $\mathrm{C}-\mathrm{C}$ stretching vibrational band at $1200-1400 \mathrm{~cm}^{-1}[25]$.

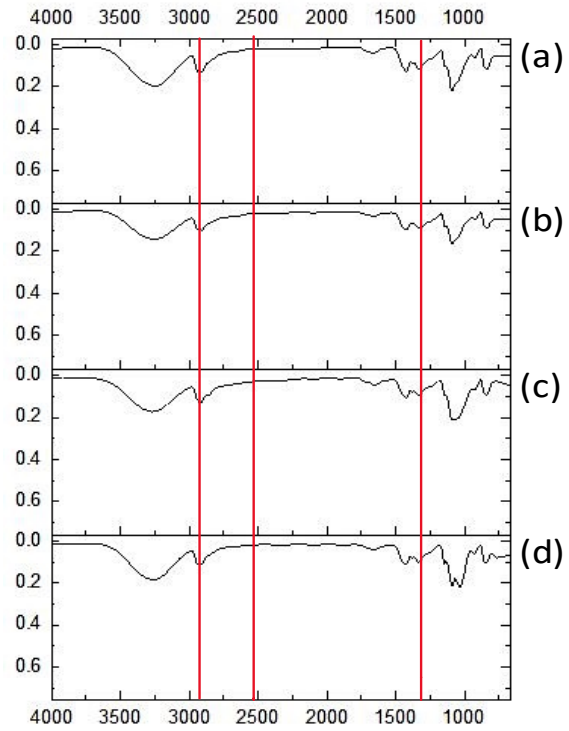

Figure 2: FT-IR spectra of lyophilized hydrogels formulations with $10 \% \mathrm{w} / \mathrm{v}$ PVA, $10 \% \mathrm{w} / \mathrm{v}$ starch and 1 crosslinking cycle: (a) Native starch, (b) 5DAS, (c) 10DAS and (d) 10DASC.

\subsection{Hydrogel Water-uptake}

Hydrogel water-uptake, or swelling ratio, is one of their most important characteristics, since this parameter is directly related to drug release mechanisms, as well as structure stability in aqueous media [1]. Water uptake capacity (\%) of the different hydrogel formulations specified in table 1 are presented in Figure 3 through 5 , where PVA composition remained constant at $10 \%$. All assays where performed at $37^{\circ} \mathrm{C}$ in $\mathrm{PBS}$ to emulate physiologic conditions. Since one of the objectives of this research was to partially substitute the weight/volume percentage of PVA in hydrogels formulations, it was necessary to compare the wateruptake percentage vs. time, according to hydrogels composed solely with PVA. In general, it was observed that 1 crosslinking cycle presented the largest swelling capacity; nevertheless, they showed the lower stability in time, with important swelling reduction toward the end of the assay. Two and three crosslinking cycles exhibited similar behaviors. At the same time, by increasing the quantity of PVA, hydrogels displayed greater stability (data not shown).

According to the analysis of variance, hydrogel formulation (both starch content and oxidation degree), number of crosslinking cycles and time had significant effects on water uptake $(p<0.05)$. Figure $3 \mathrm{a}$ shows the swelling kinetics of hydrogels with $15 \%$ starch. There were no statistical differences between native and low oxidation DAS (3, 5 and 10DAS), which displayed fast water uptake, with important fluctuations over time with one crosslinking cycle. This could be due to degradation effects that, ultimately, become relevant, as the swelling decrease after a maximum at $48 \mathrm{~h}$. When increasing the number of cycles (Figure $3 \mathrm{a}$ and $\mathrm{b}$ ), however, these fluctuations become less apparent, and decreases in water uptake are lower. These changes could indicate an improvement in hydrogel stability. In the case of 10DASC, on the other hand, had rapid swelling and seemed to reach equilibrium after $15 \mathrm{~h}$. This high oxidation DAS resulted in significantly lower swelling than the other formulations; moreover, this parameter tends to decrease with greater number of crosslinking cycles.
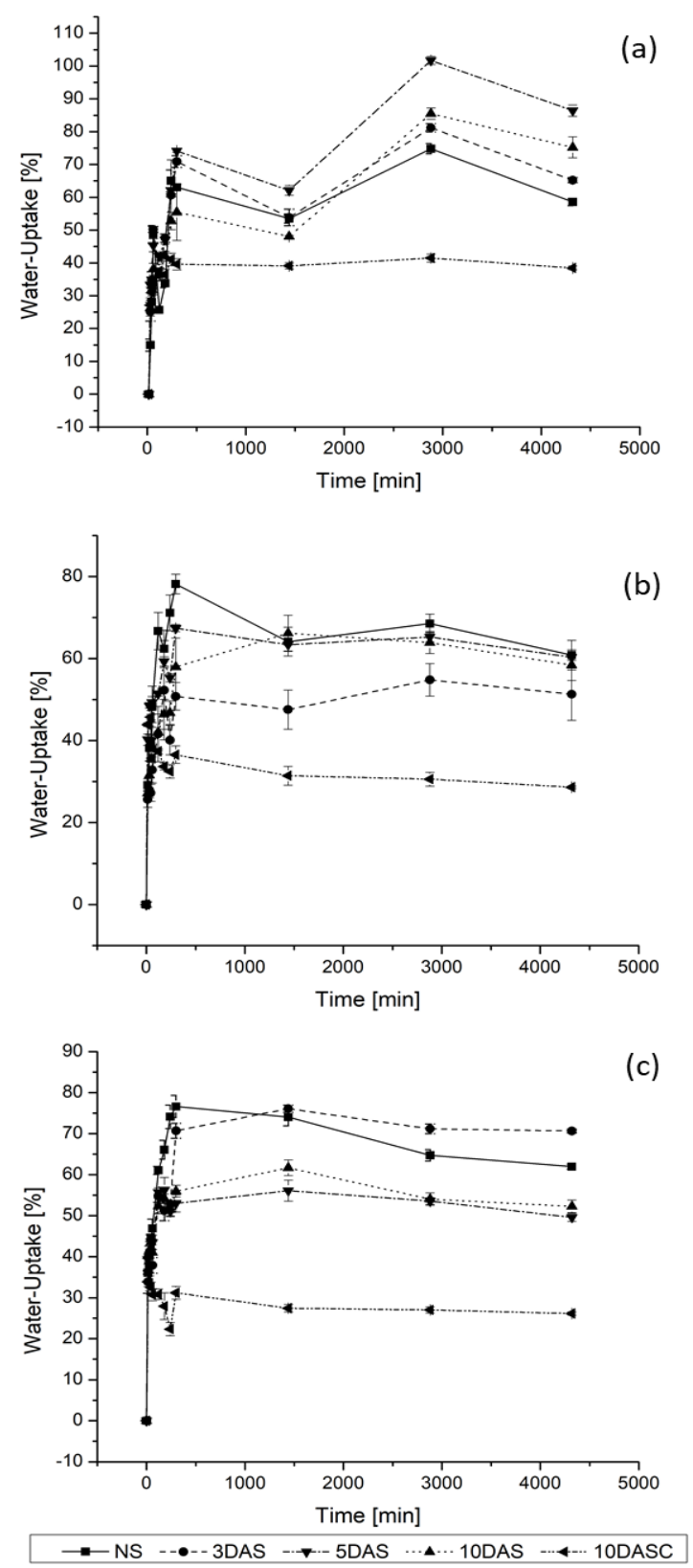

Figure 3: Swelling kinetics, expressed as water uptake percentage in time, for hydrogels composed of $10 \% \mathrm{w} / \mathrm{v}$ PVA and $15 \% \mathrm{w} / \mathrm{v}$ starch, either native (NS) of dialdehyde (3DAS, 5DAS, 10DAS and 10DASC). Formulations with (a) one crosslinking cycle, (b) two crosslinking cycles, and (c) three crosslinking cycles.

Figure 4 shows water-uptake capacity for hydrogels with $10 \%$ starch, in which, after an analysis of variance, it is determined that formulation and time affect water-uptake percentage $(p<0.05)$. Once again, the major water-uptake is obtained with one freezethaw cycle. The stability in this case is significantly reduced in comparison with Figure 3(a), where hydrogel degradation, regardless of formulation, begins at 240 min until $72 \mathrm{~h}$. After the 
statistical analysis, it was confirmed that oxidation degree had a significant effect on swelling ratio $(\mathrm{p}<0.05)$; nevertheless, there is not a clear pattern in the way the amount of carbonyl groups affects the swelling degree.
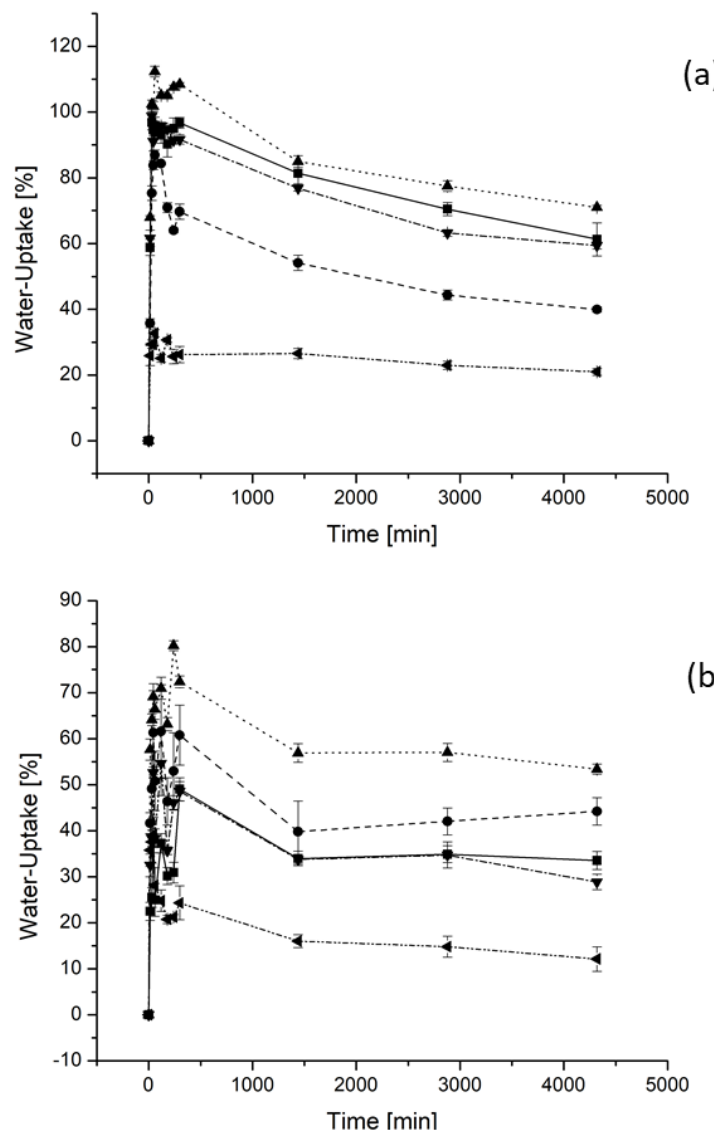

(b)

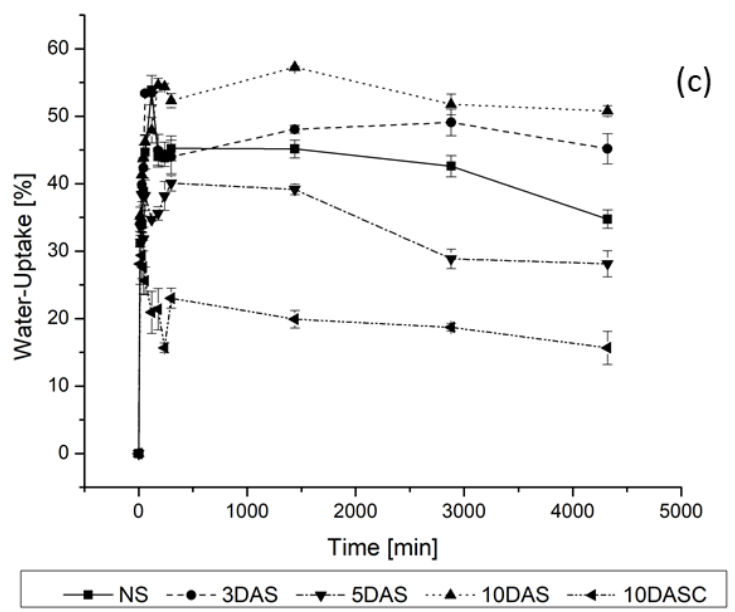

Figure 4: Swelling kinetics, expressed as water uptake percentage in time, for hydrogels composed of $10 \% \mathrm{w} / \mathrm{v}$ PVA and $10 \% \mathrm{w} / \mathrm{v}$ starch, either native (NS) of dialdehyde (3DAS, 5DAS, 10DAS and 10DASC). Formulations with (a) one crosslinking cycle, (b) two crosslinking cycles, and (c) three crosslinking cycles.

Figures 4(b) and (c) show a more accurate pattern within the effect of DAS, where starch with lower carbonyl content presents the greater swelling degree. After a Tuckey comparison on values of Figure 4(b), all the formulations are significantly different between them; nevertheless, the greatest water uptake was obtained in formulations with 3DAS and 10DAS, which agrees with lower carbonyl content. At three freeze-thaw cycles, Figure 4(c), Tukey analysis shows that there is not a difference between formulations with $3 \%$ DAS and $10 \%$ DAS. In all cases, the way time affects swelling degree, establishes an equilibrium tendency up to $72 \mathrm{~h}$. Finally, the number of cycles does affect hydrogel response, being inversely proportional to the swelling degree. However, the swelling degree within a time greater than $24 \mathrm{~h}$, is not statistically different between formulations with 2 and 3 freezethaw cycles.

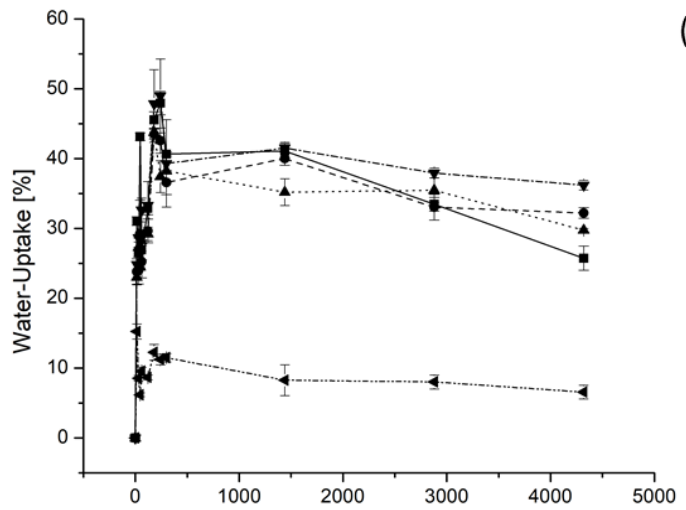

(a)

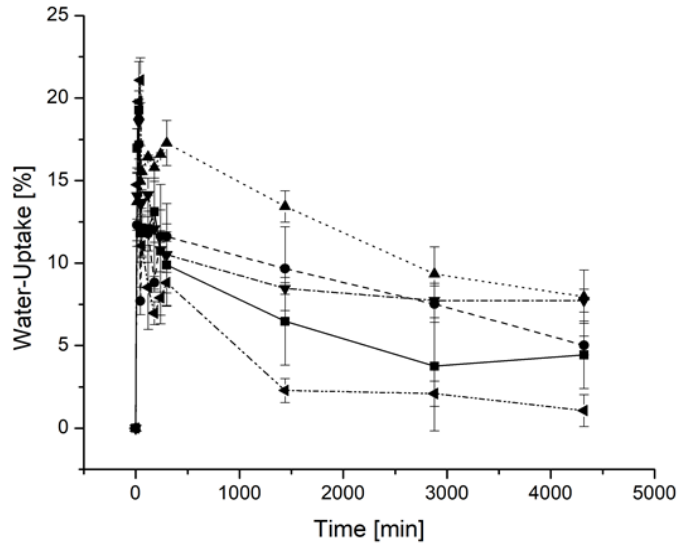

(b)

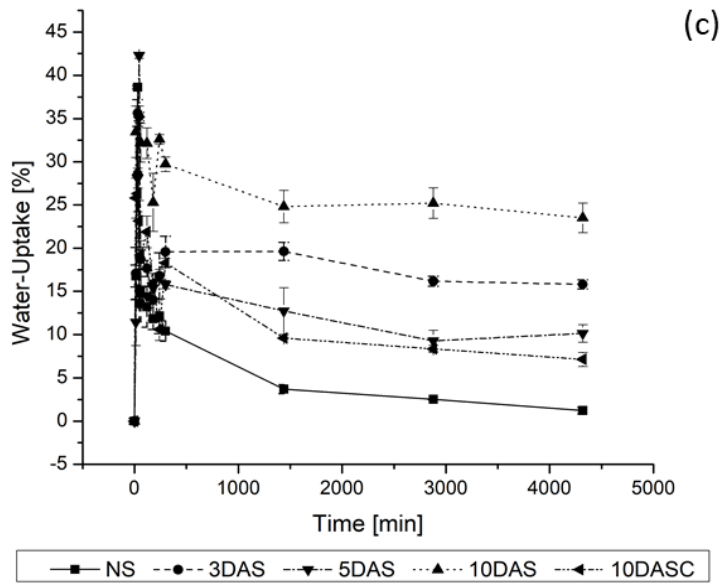

Figure 5: Swelling kinetics, expressed as water uptake percentage in time, for hydrogels composed of $10 \% \mathrm{w} / \mathrm{v}$ PVA and $5 \% \mathrm{w} / \mathrm{v}$ starch, either native (NS) of dialdehyde (3DAS, 5DAS, 10DAS and 10DASC). Formulations with (a) one crosslinking cycle, (b) two crosslinking cycles, and (c) three crosslinking cycles. 
By comparing hydrogels with $15 \%$ and $10 \mathrm{w} / \mathrm{v}$ starch (Figures 3 and 4 , respectively), there are clear differences. The greater water-uptake in the first case is presented at $300 \mathrm{~min}$; while, for the latter, this value is obtained at $180 \mathrm{~min}$. There is a major hydrogel degradation within the first $24 \mathrm{~h}$ at a lower starch content. These results suggest that oxidized starch content affects swelling.

Finally, swelling kinetics for hydrogels formulations with 5\% starch are shown in Figure 5. Considering factors such as formulations and number of cycles, swelling degree in all cases was considerably lower, compared with 15 and $10 \%$ starch (Figures 3 and 4), due to the decrease of polymer content in the structure. The statistical analysis resolved that there is not a significant variation on swelling, considering the formulation of the hydrogel as a swelling response. At this low concentration of starch, there is not a clear pattern on the effect of the oxidation degree on swelling, which could be due to a predominant effect of PVA, independent of the starch's characteristics.

Literature shows that starch oxidation affects the surface in the granule by increasing the porosity and presence of holes in the structure $[20,26]$. This could explain the reason behind a large water-uptake in hydrogels formulations with dialdehyde starch. Even if there is a significant presence of hydrophobic groups in modified starch, this porosity helps hydrogels to increase not only their swelling degree, but also their stability during a period of time.

After the statistical analysis, it was possible to show that there is a major increasing swelling degree during the first 5 hours in hydrogels with a lower carbonyl content; which agrees with previous literature reported by [27]. According to previous works, higher PVA composition would result in hydrogels with lower swelling ratio due to its higher degree of crystallinity [25]. Nonetheless, the opposite effect occurs after the addition of dialdehyde starch. In [28], the authors reported that oxidation occurs mainly in the amorphous lamella of the semi-crystalline growth rings in starch granules. They evaluated the effect of different degrees of oxidation with sodium hypochlorite on bean starch. A higher carbonyl content in dialdehyde bean starch resulted in a decrease of the crystallinity, suggesting that the amylopectin chains were damaged and therefore, there is a degradation of the crystalline region. The conclusion proposed in the current research behind the large water-uptake of hydrogels composed with PVA and dialdehyde starch, is due to the significant presence of less crystalline polymer.

An important fact about dialdehyde starch at a high oxidation degree was evidenced through a Tukey analysis. The way in which time affects swelling degree, in all cases, follows a tendency in which water uptake within the first hour is rather high. Suddenly, there is a significant de-swelling until $240 \mathrm{~min}$. Beyond this time, hydrogels exhibit stability. In [10], the authors reported that at higher carbonyl content, the crystallinity decreases, and at a certain point, starch structure becomes amorphous. It can be assumed that this de-swelling is due to the less crystallinity arrangement, even though, there is stability during times greater than $240 \mathrm{~min}$.

Finally, swelling degree is related with the porosity of hydrogels, evidenced through the scanning electron micrographs of lyophilized hydrogels. As presented in Figure 1, oxidation degree does affect the morphology of hydrogels, by allowing flatter and seemingly larger pores in the case of modified starch at a low oxidation degree. The behavior according to porosity and swelling degree is as expected, since 10DAS presents not only large porosity, but also greater water-uptake; unlike, 10DASC, which exhibits a lower porosity and so was the swelling degree with these formulations.

\subsection{Ibuprofen release}

Hydrogels have sparked interest in drug delivery applications due to their porous structures, that assists drug loading in the polymeric matrix, for a subsequent sustained release. In the present work, ibuprofen was used as a model drug that can give insights into potential applications for other non-steroidal antiinflammatory drugs (NSAIDs). In this case, control hydrogels were done at $20 \%$ PVA, since those at $10 \%$ were rather unstable and disintegrated quickly. According to ANOVA, ibuprofen release was significantly affected by composition, time and number of freeze-thaw, or crosslinking, cycles. Release assays are presented in Figure 6.

(a)
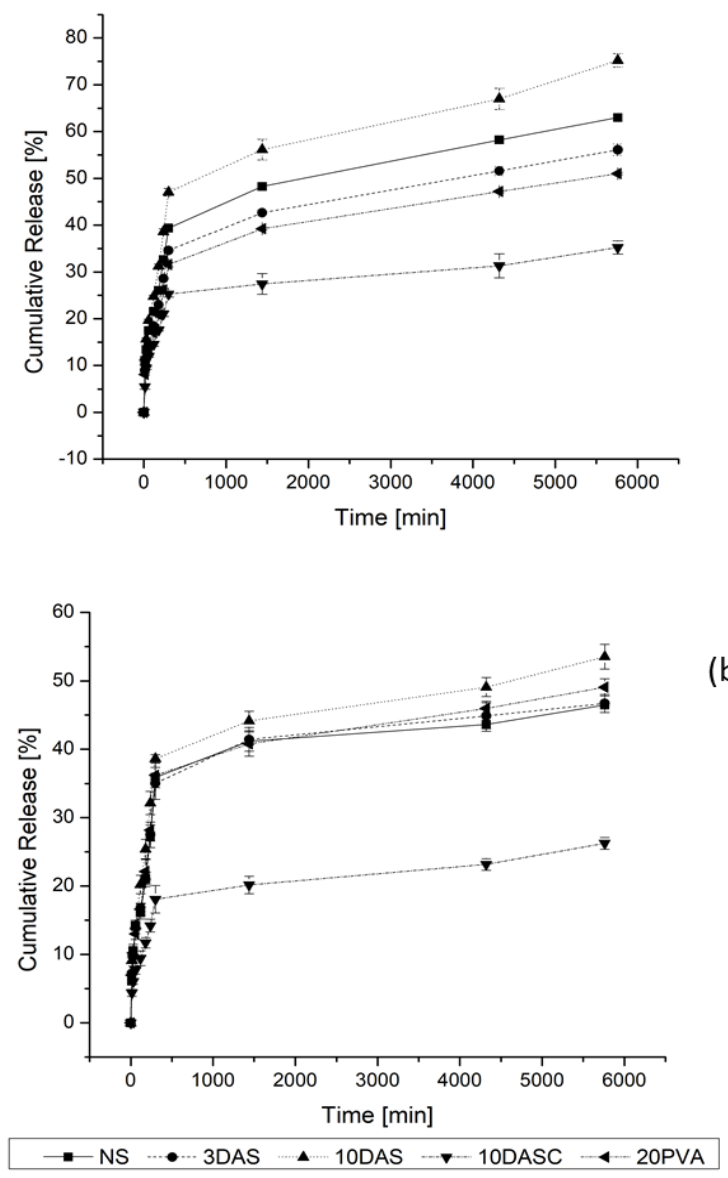

Figure 6: Ibuprofen cumulative release profiles from hydrogels with $10 \% / \mathrm{v}$ PVA and $10 \%$ starch at different oxidation levels. Only PVA at $20 \%$ was used as control. (a) One crosslinking cycle and (b) Two crosslinking cycles.

For all formulations, there was a fast release in the first 6 hours, followed by small, not statistically significant, increases, indicating a tendency toward equilibrium. In hydrogels with one crosslinking cycle (Figure 6a), there is a very similar ibuprofen 
profile in every hydrogel formulation. After Tukey comparison, it was shown that formulations with native starch, 3DAS, 10DAS and solely $20 \%$ PVA have no significant differences between them. Interestingly, hydrogels with native starch and low oxidation DAS displayed greater swelling, and some degradation that played an important role in the release. Hydrogels with 10DASC, on the other hand, did present significantly lower cumulative release at every time point. All these previous formulations exhibit the same behavior; the largest drug release was performed within the first 6 hours of contact with PBS; and, there is an equilibrium tendency at $72 \mathrm{~h}$.

Values of ibuprofen release in hydrogels with two crosslinking cycles, Figure 6b, were significantly affected by formulations and time. Again, Tukey comparisons indicated that formulations with 3DAS, 10DAS and solely $20 \%$ PVA are similar among them, representing the formulations with largest drug release percentage. Similar to what occurred with one cycle, 10DASC resulted in hydrogels with the lowest levels of release. This time, nonetheless, values of cumulative release were lower than those found with one crosslinking cycle, which relates to the lower swelling capacities found at greater number of freeze-thaw cycles. Moreover, there was a tendency to a more sustained release, possible due to the greater stability provided by more crosslinking

When comparing Figures $6 a$ and $b$, it is possible to affirm that as presented in literature, the high-water content of hydrogels results in an increased release of drugs from the polymeric matrix [3]. This is confirmed by the statistical analysis. The tendency in both cases is that hydrogels with 10DAS exhibit the largest drug release, and the lowest is attributed to those with 10DASC.

In hydrogels, the rate of drug diffusion through the polymeric structure depends on the extent of cross-linking. Converting the ensemble of individual polymer chains into a macromolecular network allows the water diffusion into the interstices of the network. Comparing Figures $6 a$ and $b$, there is indication that the extent of cross-linking minimizes the swelling and, thereby, the diffusion of the drug. The reason behind this behavior is due to the fact that ibuprofen is an entrapped drug, and the rate of diffusion depends on the interchain separation and the size of the diffusing drug [29].

\section{Conclusions}

The oxidation degree of cassava starch has important effects on the properties of hydrogels with PVA, particularly at larger carbonyl contents. At a low oxidation degree obtained with $10 \%$ $\mathrm{v} / \mathrm{v}_{2} \mathrm{O}_{2}$ and $3 \% \mathrm{v} / \mathrm{v}_{2} \mathrm{O}_{2}$, hydrogels showed the major suitable characteristics of hydrogels for drug delivery applications, since the water-uptake percentage was higher, as it was drug release. Considering hydrogels formulations with DAS catalyzed with copper sulfate (II), and the largest carbonyl content, there was a significant reduction of water-uptake through the polymeric structure, due to strong intermolecular bonds, which resulted in a reduced swelling degree, but, as expected, those showed the major stability during time, proving an improved stability in physiological environment. It is possible that, for the latter formulation, the hydrogels could be more appropriate for tissue engineering because, given that they do not swell significantly, their mechanical properties could be superior to those that presented higher swelling ratios. Additionally, being more stable in time, they could potentially meet mechanical demands for longer periods, and we recommend doing this assessment in future studies.

\section{Conflict of Interest}

The authors declare no conflict of interest.

\section{Acknowledgment}

This work was funded by the PoliGrant program at Universidad San Francisco de Quito. Publication of this article was funded by the Academic Articles Publication Fund of Universidad San Francisco de Quito USFQ

\section{References}

[1] A.S. Hoffman, "Hydrogels for biomedical applications," Advanced Drug Delivery Reviews, 64(SUPPL.), 18-23, 2012, doi:10.1016/j.addr.2012.09.010.

[2] J.L. Holloway, A.M. Lowman, G.R. Palmese, "The role of crystallization and phase separation in the formation of physically cross-linked PVA hydrogels," Soft Matter, 9(3), 826-833, 2013, doi:10.1039/c2sm26763b.

[3] T.R. Hoare, D.S. Kohane, "Hydrogels in drug delivery: Progress and challenges," $\quad$ Polymer, 49(8), 1993-2007, 2008 doi:10.1016/j.polymer.2008.01.027.

[4] E.A. Kamoun, "N-succinyl chitosan-dialdehyde starch hybrid hydrogels for biomedical applications," Journal of Advanced Research, 7(1), 69-77, 2016, doi:10.1016/j.jare.2015.02.002.

[5] S. Jiang, S. Liu, W. Feng, "PVA hydrogel properties for biomedical application," Journal of the Mechanical Behavior of Biomedical Materials, 4(7), 1228-1233, 2011, doi:10.1016/j.jmbbm.2011.04.005.

[6] Y. Zhu, C. Romain, C.K. Williams, "Sustainable polymers from renewable resources," Nature, 540(7633), 354-362, 2016, doi:10.1038/nature21001.

[7] H. Hu, F.J. Xu, "Rational design and latest advances of polysaccharide-based hydrogels for wound healing," Biomater Sci., 8(8), 2084-2101, 2020, doi:10.1039/d0bm00055h.

[8] A.R.G. Dias, E.D.R. Zavareze, E. Helbig, F.A. De Moura, C.G. Vargas, C.F. Ciacco, "Oxidation of fermented cassava starch using hydrogen peroxide," Carbohydrate Polymers, 86(1), 185-191, 2011, doi:10.1016/j.carbpol.2011.04.026.

[9] Y. Dou, B. Zhang, M. He, G. Yin, Y. Cui, I.N. Savina, "Keratin/polyvinyl alcohol blend films cross-linked by dialdehyde starch and their potential application for drug release," Polymers, 7(3), 580-591, 2015, doi:10.3390/polym7030580.

[10] Y.R. Zhang, X.L. Wang, G.M. Zhao, Y.Z. Wang, "Preparation and properties of oxidized starch with high degree of oxidation," Carbohydrate Polymers, 87(4), 2554-2562, 2012, doi:10.1016/j.carbpol.2011.11.036.

[11] V.M. de Oliveira Cardoso, B. Stringhetti Ferreira Cury, R.C. Evangelista, M.P. Daflon Gremião, "Development and characterization of cross-linked gellan gum and retrograded starch blend hydrogels for drug delivery applications," Journal of the Mechanical Behavior of Biomedical Materials 65, 317-333, 2017, doi:10.1016/j.jmbbm.2016.08.005.

[12] A.P. Erazo Bastidas, J. Alvarez Barreto, Synthesis and Characterization of Hydrogels based on Poly ( vinyl alcohol ) and Modified Starch as Carriers for Controlled Drug Delivery, Quito, 2018

[13] Y.-R. Zhang, X.-L. Wang, G.-M. Zhao, Y.-Z. Wang, "Influence of oxidized starch on the properties of thermoplastic starch," Carbohydrate Polymers, 36(1), 358-364, 2013, doi:10.1016/j.carbpol.2013.03.093.

[14] Y. Yu, Y. Wang, W. Ding, J. Zhou, B. Shi, "Preparation of highly-oxidized starch using hydrogen peroxide and its application as a novel ligand for zirconium tanning of leather," Carbohydrate Polymers, 174, 823-829, 2017, doi:10.1016/j.carbpol.2017.06.114.

[15] P. Parovuori, A. Hamunen, P. Forssell, K. Autio, K. Poutanen, "Oxidation of Potato Starch by Hydrogen Peroxide,” Starch - Stärke, 47(1), 19-23, 1995, doi:10.1002/star.19950470106

[16] Y. Lu, Q.M. Kong, R. Jing, X. Hu, P.X. Zhu, "Solid state oxidation of polyvinyl alcohol by hydrogen peroxide-Cu (II)," Polymer Degradation and $\begin{array}{lll}\text { Stability, } & \mathbf{9 8}(6), & 1103-1109,\end{array}$ doi:10.1016/j.polymdegradstab.2013.03.022.

[17] R.J. Smith, Characterization and analysis of starches, Academic Press, New 
York and London: 620-625, 1967.

[18] P.M. Castro, P. Fonte, A. Oliveira, A.R. Madureira, B. Sarmento, M.E. Pintado, "Optimization of two biopolymer-based oral films for the delivery of bioactive molecules," Materials Science and Engineering C, 76, 171-180, 2017, doi:10.1016/j.msec.2017.02.173.

[19] K. Sangseethong, N. Termvejsayanon, K. Sriroth, "Characterization of physicochemical properties of hypochlorite- and peroxide-oxidized cassava starches," Carbohydrate Polymers, 82(2), 446-453, 2010, doi:10.1016/j.carbpol.2010.05.003.

[20] L.M. Fonseca, J.R. Gonçalves, S.L.M. El Halal, V.Z. Pinto, A.R.G. Dias, A.C. Jacques, E. da R. Zavareze, "Oxidation of potato starch with different sodium hypochlorite concentrations and its effect on biodegradable films," LWT - Food Science and Technology, 60(2), 714-720, 2015, doi:10.1016/j.lwt.2014.10.052.

[21] Q.L. Loh, C. Choong, "Three-dimensional scaffolds for tissue engineering applications: Role of porosity and pore size," Tissue Engineering - Part B: Reviews, 19(6), 485-502, 2013, doi:10.1089/ten.teb.2012.0437.

[22] O. Moreno, J. Cárdenas, L. Atarés, A. Chiralt, "Influence of starch oxidation on the functionality of starch-gelatin based active films," Carbohydrate Polymers, 178, 147-158, 2017, doi:10.1016/j.carbpol.2017.08.128.

[23] E.R. Kenawy, E.A. Kamoun, M.S. Mohy Eldin, M.A. El-Meligy, "Physically crosslinked poly(vinyl alcohol)-hydroxyethyl starch blend hydrogel membranes: Synthesis and characterization for biomedical applications," Arabian Journal of Chemistry, 7(3), 372-380, 2014, doi:10.1016/j.arabjc.2013.05.026.

[24] A.L. Da Róz, M.D. Zambon, A.A.S. Curvelo, A.J.F. Carvalho, "Thermoplastic starch modified during melt processing with organic acids: The effect of molar mass on thermal and mechanical properties," Industrial Crops and Products, 33(1), 152-157, 2011, doi:10.1016/j.indcrop.2010.09.015.

[25] S.C. Pang, S.F. Chin, S.H. Tay, F.M. Tchong, "Starch-maleate-polyvinyl alcohol hydrogels with controllable swelling behaviors," Carbohydrate Polymers, 84(1), 424-429, 2011, doi:10.1016/j.carbpol.2010.12.002.

[26] D. Kuakpetoon, Y.J. Wang, "Locations of hypochlorite oxidation in corn starches varying in amylose content," Carbohydrate Research, 343(1), 90100, 2008, doi:10.1016/j.carres.2007.10.002.

[27] A. V. Reis, M.R. Guilherme, T.A. Moia, L.H.C. Mattoso, E.C. Muniz, E.B. Tambourgi, "Synthesis and characterization of a starch-modified hydrogel as potential carrier for drug delivery system," Journal of Polymer Science Part A: Polymer Chemistry, 46(7), 2567-2574, 2008, doi:10.1002/pola.22588

[28] N.L. Vanier, E. Da Rosa Zavareze, V.Z. Pinto, B. Klein, F.T. Botelho, A.R.G. Dias, M.C. Elias, "Physicochemical, crystallinity, pasting and morphological properties of bean starch oxidised by different concentrations of sodium hypochlorite," Food Chemistry, 131(4), 1255-1262, 2012, doi:10.1016/j.foodchem.2011.09.114.

[29] L. Djekic, M. Martinović, V. Dobričić, B. Čalija, Đ. Medarević, M. Primorac, "Effect of Bioadhesive Polymers on Stability and Drug Release Kinetics of Biocompatible Hydrogels for Topical Application of Ibuprofen.," J Pharm Sci., 108(3), 1326-1333, 2019, doi:10.1016/j.xphs.2018.10.054. 